\title{
NICE thoughts?
}

\section{Derek Richards}

\section{Director, Centre for Evidence-based Dentistry, Oxford, UK}

The guidance from the National Institute of Clinical Effectiveness (NICE) on third molars has provoked a debate. We take a look at the evidence behind this guidance and the guidelines produced by the Scottish Intercollegiate Guidelines Network (SIGN).

The recently published guidelines from the National Institute of Clinical Effectiveness (NICE) on the removal of third molars (http://server1.nice.org.uk/niceweb/Cat.asp?c=509), have caused considerable debate. In this issue we take a look at the review conducted by the Centre for Reviews and Dissemination at York which formed part of the evidence behind these guidelines. We also look at the guidelines produced by the Scottish Intercollegiate Guidelines Network (SIGN) on the same topic (http:// www.sign.co.uk). Both essentially cover the same ground, but from a different perspective. Why is this?

One of the essential elements of evidence-based dentistry is that the best available evidence should be combined with clinical experience, and this should be applied to the clinical problem with which the dental practitioner is faced. The NICE guideline process had been attacked because no dentists were involved in the appraisal and final production of the guideline. On the one hand, it could be argued that this has produced a more objective review of the evidence, because the NICE panel should be free of clinical biases. On the other it could be argued that without the clinical experience they couldn't assess the evidence, particularly if the overall quality is poor, as is the case in this review.
When comparing this with the SIGN process, which had input from a large clinical dental panel, a subtly different focus emerges. These differences serve to strengthen the point that we all need the skills of critical appraisal to have an objective debate about the evidence. Critical appraisal of evidence is an essential skill for the modern practitioner, a point that we will continue to stress in this journal. In this issue we also outline the process of critical appraisal for our readers. Future issues will provide readers with information on how to critically appraise different types of studies.

The NICE process has also been challenged by some for the apparent lack of a clear and open process in relation to other reviews. Recently there was an interesting discussion regarding this on the evidence-based health care mailing list. It is clear that, as a new organisation, NICE will need to develop and improve its structures and systems. If this is allowed to happen, NICE can become an important agent for improving health care provision in the UK.

The NICE guidance was also challenged as it was said not to tell the profession anything it did not already know. However it is often helpful to remind clinicians of the need to change practice. Are we really all following the guidelines for removal of wisdom teeth? Clear indications for the removal of wisdom teeth have been around for many years, with one of the first formalised guidelines coming after a National Institutes of Health Consensus Conference in $1979^{1}$. One of the goals of NICE is to deal with variations in health care provision, and it would be a brave person who argued that there were no differences in the intervention rates for the removal of wisdom teeth within the profession.

One of the common problems associated with wisdom teeth in particular, and dentistry in general, is pain. The perception of pain is subjective and, despite a large amount of research into pain relief, it is often not well managed. This issue addresses the problems of systematic reviews in pain with an editorial from Andrew Moore at the Oxford Pain Unit. We also look at three systematic trials of the more common non-steroidal anti-inflammatory drugs (NSAIDs) available for pain relief. These come from the pain literature, which may be unfamiliar to many dentists. However, the humble third molar is frequently the clinical model chosen to test analgesics. We also take a look at the detailed listing of the systematic reviews available on the Oxford Pain database.

1. NIH consensus development conference for removal of third molars. J Oral Surg 1980; 38: 235-236. 